\title{
An Empirical Exponential Model based on Reflectivity Measurements for Soil Nitrogen Detection
}

\author{
Deepti Lourembam \\ Department of Electronics and Communication \\ Engineering, \\ Assam Don Bosco University \\ Azara, Assam
}

\author{
Subra Mukherjee \\ Department of Electronics and Communication \\ Engineering, \\ Assam Don Bosco University \\ Azara, Assam
}

\begin{abstract}
Right amount of nutrients in soil plays an important role in maintaining the health of the plant as well as the soil. In this regard, developing novel ways of soil macronutrient detection has become a must in the present day scenario. Studies based on diffused reflectance for detecting soil nutrients and attributes have taken great strides towards building up more effective systems. These trends have been contributing significantly to precision farming. Reflectance measurements in the near-infrared region have proved to be reliable in terms of measuring various soil attributes like $\mathrm{pH}$, organic matter and soil nutrients. In the current work, a system was designed using an NIR source of $850 \mathrm{~nm}$ to detect the amount of nitrogen present in soil. It was used to collect the reflectivity of the sample when variable amount of chemical was added which was then correlated to the output voltage. Based on the raw data collected, a mathematical model was developed through statistical analysis. The exponential based model was found to have the best fit with the characteristic data obtained from the sensor when analyzed statistically. The model performance estimated for the optimized combination were $\mathrm{R}^{2}$ of 0.99 and RMSE of 0.5. Moreover, an algorithm based on this model was tested and validated with a success rate of 90\%. Based on the thresholds obtained from experimentation, an Arduino was programmed in order to detect the presence of nitrogen in soil as low, medium and high. The system so designed can be employed as a cost-effective optical sensor for detection of soil nitrogen and can make a significant impact on precision farming.
\end{abstract}

\section{General Terms}

Optical sensors, Precision farming, Nitrogen, Vis-NIR Spectroscopy, Root mean Square error, Coefficient of determination, Regression Analysis, Error sum of Square, Total Sum of Square, Reflectivity

\section{Keywords}

Precision farming, mathematical modeling, Nitrogen, Sensors, Reflectivity, Soil Nutrients.

\section{INTRODUCTION}

Precision farming is earning a big name in the field of agriculture with newer trends taking over the conventional ones over the following years. Precise and accurate measurements of the variability within the field is important for site-specific crop management. The variability is affected by factors like soil properties, nutrients and crop yield. Sensing techniques can give an overall idea about the data required for the right amount of inputs. Ideally sensor devices are fitted with a global positioning system to allow for soil data to be captured on-the-go and instantaneously converted into distribution maps. This will help in real-time monitoring and intervention of soil nutrient. [1]. The majority of the soil sensors described such as mechanical, electrochemical and optical sensor provides output that is affected by more than one soil characteristics. Precision and accuracy have been used for assessment of sensor performance [2]. Optical sensors are currently employed in various fields and have diverse applications. Optical components like LEDs have the advantage of comparatively smaller size which gives an edge when designing portable systems. One of the most interesting application of optical sensors is in the field of soil science. The use of optical properties like reflectance to detect and determine the soil physical and chemical attributes have resulted in reliable and efficient prototype sensor systems. Moreover, different soil attributes respond differently to different light sources. Most of the works reported the use of infrared light sources. Visible and near infrared spectroscopy has been extensively used in studying soil properties. The various soil attributes like texture, soil moisture, organic matter has been studied using near infrared sources. VisibleNIR spectroscopy is a rapid and non-destructive analytical technique that correlates diffusely reflected near-infrared radiation with the chemical and physical properties of materials. In [3], the potential of VIS/NIR diffuse reflectance for studying soil properties was described. The relationships between soil properties and reflectance spectra and the sources of variability in the reflectance spectra was studied in the range 250-2500nm.In [4], VIS/NIR spectroscopy was found to be reliable for prediction of $\mathrm{pH}$ value and $\mathrm{OM}$ content. When a sample is illuminated with light, certain bonds vibrate with varying electric field and the vibrations absorbs optical energy and causes light to reflect. Although optical diffused reflectance studies based on NIR spectroscopy has been used for estimation of soil organic matter, it can be extended for the study of other soil properties. The predominant absorbers in the NIR region are the $\mathrm{C}-\mathrm{H}, \mathrm{N}-\mathrm{H}$ and $\mathrm{O}-\mathrm{H}$ functional groups, making it ideal for the prediction of forms of carbon, nitrogen and water. The amount of light reflected carries useful information about the substance present in soil since a part of the light will be absorbed. In [5], near infrared spectroscopy was used to study the detection methods for nitrogen, phosphorous and Potassium along-side soil organic matter which has an important relation with nitrogen. Principle component analysis and LS-SVM was used to build up the prediction model with the coefficient of determination 0.87 for soil organic matter, 0.72 for soil available nitrogen and 0.68 for available potassium. Studies reveal that the amount of light absorbed will be different for different attributes. Soil is not only important for plant growth but it forms the very base for survival. The major macronutrients, no doubt plays an important role in giving a higher yield and improving the quality of produce. The management of these nutrients is conventionally is done by applying fertilizers. Right amount of fertilizer inputs play an important role in effective yield 
however, overutilization have adverse effects on plant growth and environment. Hence, there is a need to optimize the fertilization, increase the efficiency of crop yield as well as side effects due to leaching. Pollution of soil as well as water bodies due to chemical wash-offs are common in recent times. Moreover, when plants stores this nutrients in excess amounts, it affects the growth and appearance. Soil $\mathrm{pH}$ mapping using an on-the-go soil $\mathrm{pH}$ sensor was successfully carried out. Preceding tests under controlled conditions demonstrated a high degree of linear relationship between standard laboratory soil $\mathrm{pH}$ values and sensor $\mathrm{pH}$ values. Further investigations are necessary to improve the calibration function using additional sensor information as co-variables, such as soil reflectance, soil temperature, and soil humidity [6]. In fact, soil testing is of utmost importance since it will give a status of the overall soil availability and recommendation on fertilizer needs so that it will compliment soil nutrients for better yield keeping the soil fertility and quality intact. Precision agriculture has taken great strides since it involves dividing a field into smaller localized areas and giving the required and known amount of inputs like seeds and fertilizers. It involves optimization of plant needs taking into consideration the environmental factors. Some models developed so far takes into account the nitrogen mineralization, soil moisture content. In the case of $\mathrm{N}$ mineralization, the main purpose of modeling is to obtain quantitative data to recommend nitrogen addition. To build up the most effective model that is closest to any one of the already described existing models, the determination coefficient $\left(\mathrm{R}^{2}\right)$, the adjusted coefficient of determination (adjusted $\mathrm{R}^{2}$ ), the residual mean of squares (RMS), the residual standard deviation (RSD) were considered. Based on the results obtained in this study and on the numerical and graphical analysis of the models, the simple exponential model proposed by JONES (1984) was found to be the best model that describes the mineralization process and the potential of organic $\mathrm{N}$ to be released to the environment and to become available for plants [7]. Soil moisture based models have also been reported. A similar work has been reported in [8]. Many empirical model has been developed to describe the soil water characteristic curve. Results were compared with the most widely used models to assess the performance of the system. It was found that the modified exponential model exhibited the best fit. The curve-fitting routine describes the best possible way in which the mathematical function passes as closely to the data points. Indicators like RMSE (Root Mean Square Error) and $\mathrm{R}^{2}$ (Coefficient of Determination) was used for error analysis since it as a measure of the fit quality. In [9], a relationship between soil moisture content and bare reflectance measurements has been established and the intrinsic performance has been estimated by computing the standard deviation $\sigma$ and the coefficient of determination $\mathrm{R}^{2 .}$ The soil model performance shows that, among all of the a priori classes, $\mathrm{R}^{2}$ is better than $97 \%$ with $\sigma$ lower than 0.01 . The quality of the SMC estimation is assessed by computing the $\mathrm{R}^{2}$ and the RMSE.A similar study was made where the properties of soil organic properties were studied using fractal based feature extraction. The model accuracies were evaluated on estimated and measured soil SOC, $\mathrm{N}$ and $\mathrm{pH}$ values using RMSE, $\mathrm{R}^{2}$ and the ratio of percent deviation. The first 30 PCA components were combined with fractal features and then ingested into the gradient-boosting regression model. Combined features showed better performance when applied for the estimation of all three soil properties, SOC $\left(\mathrm{R}^{2}=0.86\right.$, $\mathrm{RMSE}=55.16 \mathrm{~g} / \mathrm{kg}, \mathrm{RPD}=2.7), \mathrm{N}\left(\mathrm{R}^{2}=0.78, \mathrm{RMSE}=2.96 \mathrm{~g} / \mathrm{kg}\right.$, $\mathrm{RPD}=2.19)$ and $\mathrm{pH}\left(\mathrm{R}^{2}=0.85, \mathrm{RMSE}=0.44, \mathrm{RPD}=2.59\right)$ [10]. The error performance analysis is important for building up robust systems. It describes the ability of the system to adapt to same system conditions. In other words, factors like precision becomes a prime indicator while designing a good sensor system. . Remote sensing techniques are being used in agriculture. A review is reported in [11]. The use of information collected from reflectance data was described. The importance of soil nutrient assessment is highlighted. In this way, the producer can apply the inputs in the precise location and quantity they are needed for maximum yield. With recent advances in sensor technology, various techniques which are used to test the variability in soil nutrients have been developed. There has been a greater use of variable rate applicators for better crop management which promotes precision farming. Variable rate nitrogen fertilization (VRF) improves the nutrient use efficiency and helps in mitigating environmental problems. The efficiency of VRF was influenced by climatic factors. Also, a comparison was made between the conventional single rate nitrogen fertilization [12].Several studies are being made for specific crops like tea regarding their soil nutrient needs on a larger scale. The data so available can be used to build up sensor systems which can directly influence precision farming in a big way. In [13], thirty composite soil samples were randomly collected from the top soil $(0-20 \mathrm{~cm})$ in some selected tea growing soils .Several analysis were carried out to find the physio-chemical properties and total concentration of $\mathrm{N}, \mathrm{P}$ and $\mathrm{K}$. Three type of soil were identified though the analysis indicating that soil texture and type is one of the primary attribute to be considered while designing systems. The soil type was found to be acidic. One very important finding is that there was positive correlation between nitrogen and $\mathrm{pH}$ with $\mathrm{r}=0.75$. Another work reported the development of soil sensor specific for paddy field. One important feature was the use of NIR and visible spectroscopy real-time [14]. In [15], optical sensors were used to measure nitrogen status in cotton. The experiments were carried out around four wavelength range in near infrared region using the reflectance from the plant. The application of such sensor system will probably compliment the concept of precision farming or site-specific crop management where the variability within a particular field is studied by segmenting the field into localized areas.

A lot of work has been currently focused on designing systems that detects soil macronutrients with better precision since the present day scenario shows a greater part of crop production being disturbed by imbalance in soil nutrients caused due to over-utilization of fertilizers. Hence, there is an ardent need to design cost-effective sensors which will contribute significantly for recommending right amount of inputs for particular plant taking care of environmental factors.

\section{DESCRIPTION OF THE DATABASE AND RELATED MODEL}

After several extensive experiments based on different combinations, a model was proposed in this work. It is based on the soil reflectivity depending on the soil nitrogen content. The characteristic curve is presented in this section.

\subsection{The developed database}

The database was formed from over 200 combinations resulting from the sub-samples of the same soil type collected from a particular field. It was collected through grid sampling. The soil type was to be clayey-loam type. 


\subsection{Experimental procedure and measurement method}

Soil reflectivity was measured using the diffused reflectance method. Each sample which was air-dried was kept in a beaker. Soil samples were then mixed with Urea and ammonium hydroxide which acts as the nitrogen source. Distilled water was used so as to maintain the $\mathrm{pH}$. A nearinfrared source of $850 \mathrm{~nm}$ and detector was used. Several combinations of soil and chemical was mixed to study the response of the sample. After obtaining the response, a trend was established. The experiments has been carried out in the dark room.

\subsection{Model Analysis}

Regression analysis was carried out in order to establish the relation between the independent variable and the dependent variable. In this context, it can be used to find the correlation between the soil nitrogen content and the reflectivity of soil samples. After which optimization techniques are used to obtain the best fit parameters for soil-nitrogen characteristic curves. The Soil-Nitrogen characteristics curve can be defined as the relationship between soil nitrogen content w.r.t the reflectivity. The characteristics curve or trend follows an exponential decay describing the reflectivity when subjected to various soil nitrogen content. In order to further sharpen the model so developed, statistical tools can be used to analyze the model behavior so as to check whether the system is free from error and accuracy of the system in terms of error performance can be verified. For accessing the quality of the proposed model, accurately studying the prediction error is of utmost importance. However, some techniques available may create misleading results which might lead to over-fitting the data. The concept behind over-fitting comes into picture when the training data is fitted very well but the model fails when new data-sets are used as test data. The main aim should be to build up a model that predicts well for new data-sets used for testing. Preventing over-fitting and under-fitting is the key to build up robust and reliable systems. In this study, the rootmean-square error (RMSE) and the coefficient of determination $\left(\mathrm{R}^{2}\right)$ was used to study the system performance. The RMSE indicates the overall error of the evaluated model function with an ideal value close to 0 . The coefficient of determination indicates the fit quality. It describes the variability of the dependent variable based on the variability of the independent variable. The ideal value is close to 1 . It is one of the widely used tools in error analysis of a system. The RMSE can be expressed as:

$$
\begin{gathered}
R M S E=\sqrt{\frac{\mathbf{1}(\boldsymbol{S S E})}{\boldsymbol{N}}} \\
S S E=\sum_{1}^{N}\left(y_{i}^{o b s}-y_{i}^{f i t}\right)^{2}
\end{gathered}
$$

The coefficient of determination, $\mathrm{R}^{2}$ can be expressed as:

$$
R^{2}=1-\frac{S S E}{S S T}
$$

Where $\mathrm{SSE}=$ error sum of squares

$$
\text { SST=Total sum of squares }
$$

Where $\boldsymbol{y}_{\boldsymbol{i}}^{\boldsymbol{o b s}}=$ Observed experimental data

$$
\begin{aligned}
& \boldsymbol{y}_{\boldsymbol{i}}^{\boldsymbol{f i t}}=\text { Predicted data } \\
& \mathrm{N}=\text { Number of observations }
\end{aligned}
$$

Table 1. Error performance Analysis

\begin{tabular}{|c|c|c|c|}
\hline EXPERIMENT & SSE & \multicolumn{1}{|c|}{ RMSE } & $\mathbf{R}^{2}$ \\
\hline 1 & 8.48 & 1.3 & 0.994 \\
\hline 2 & 23.2 & 2.17 & 0.989 \\
\hline 3 & 1.283 & 0.5 & 0.999 \\
\hline 4 & 3.31 & 0.8 & 0.998 \\
\hline 5 & 7.72 & 1.2 & 0.996 \\
\hline
\end{tabular}

Exponential regression analysis was performed to fit the experimental data into the best fitted curve. The system was then represented mathematically through this model. It was based on the reflectivity values as obtained from the experiments. The error performance analysis in terms of $\mathrm{R}^{2}$ and RMSE for the exponential model analyzed as per reflectivity is shown in table 1 .

\subsection{Calibration of the system}

A general equation representing the system was established through exponential regression analysis. For every combination, it was observed that the constant values were unique. If all the parameters are known, we can calculate ' $x$ ' given the value of ' $y$ '. From the above table 1, the RMSE of experiment 3 is optimal since its value closest to 0 and $R^{2}$ was close to 1 . Thus, this combination was seen to have the optimal value. For further analysis and calibration of the system, $\ln (\mathrm{y} / \mathrm{A})=\ln \left(\mathrm{e}^{-\mathrm{b} x}\right)$

$\ln \left(\frac{y}{A}\right)=-b x$

\begin{tabular}{|c|c|c|c|c|}
\hline $\begin{array}{c}\text { SOIL } \\
\text { AMOUNT } \\
\text { (in grams) }\end{array}$ & $\begin{array}{l}\text { OUTPUT } \\
\text { VOLTAGE, y } \\
\text { (in } \mathrm{mV} \text { ) }\end{array}$ & A & b & $\begin{array}{l}\text { CHEMICAL } \\
\text { AMOUNT } \\
\text { (in grams) }\end{array}$ \\
\hline 40 & 140.3 & 157.4 & 0.03 & 3.8 \\
\hline 40 & 123.4 & 157.4 & 0.03 & 8.12 \\
\hline 40 & 110.6 & 157.4 & 0.03 & 11.7 \\
\hline 40 & 98.2 & 157.4 & 0.03 & 15.7 \\
\hline 40 & 86.9 & 157.4 & 0.03 & 19.8 \\
\hline
\end{tabular}

$x=\ln (y / A) /(-b)$

Where $\mathrm{A}$ is the initial value of $\mathrm{y}$ when $\mathrm{x}$ is 0 and $\mathrm{b}$ is the rate of decay.

Table 2. Calibration of the System

The optimized 40g combination was considered for calibration of the system. Random values of output voltage, ' $y$ ' were assigned which was already obtained in the database. The value of ' $x$ ' was calculated which will ultimately determine the amount of nitrogen as low, medium or high. It was necessary to verify whether the model works for random values of $y$. This test was carried out for almost 90 data points and the success rate was found to be $90 \%$. The main importance of this analysis is to check whether the new data fits into the model as test datasets. This was so as to prevent over-fitting. Table 2 shows the calibration of the system. Figure 1 shows the exponential model based on soil reflectivity. 

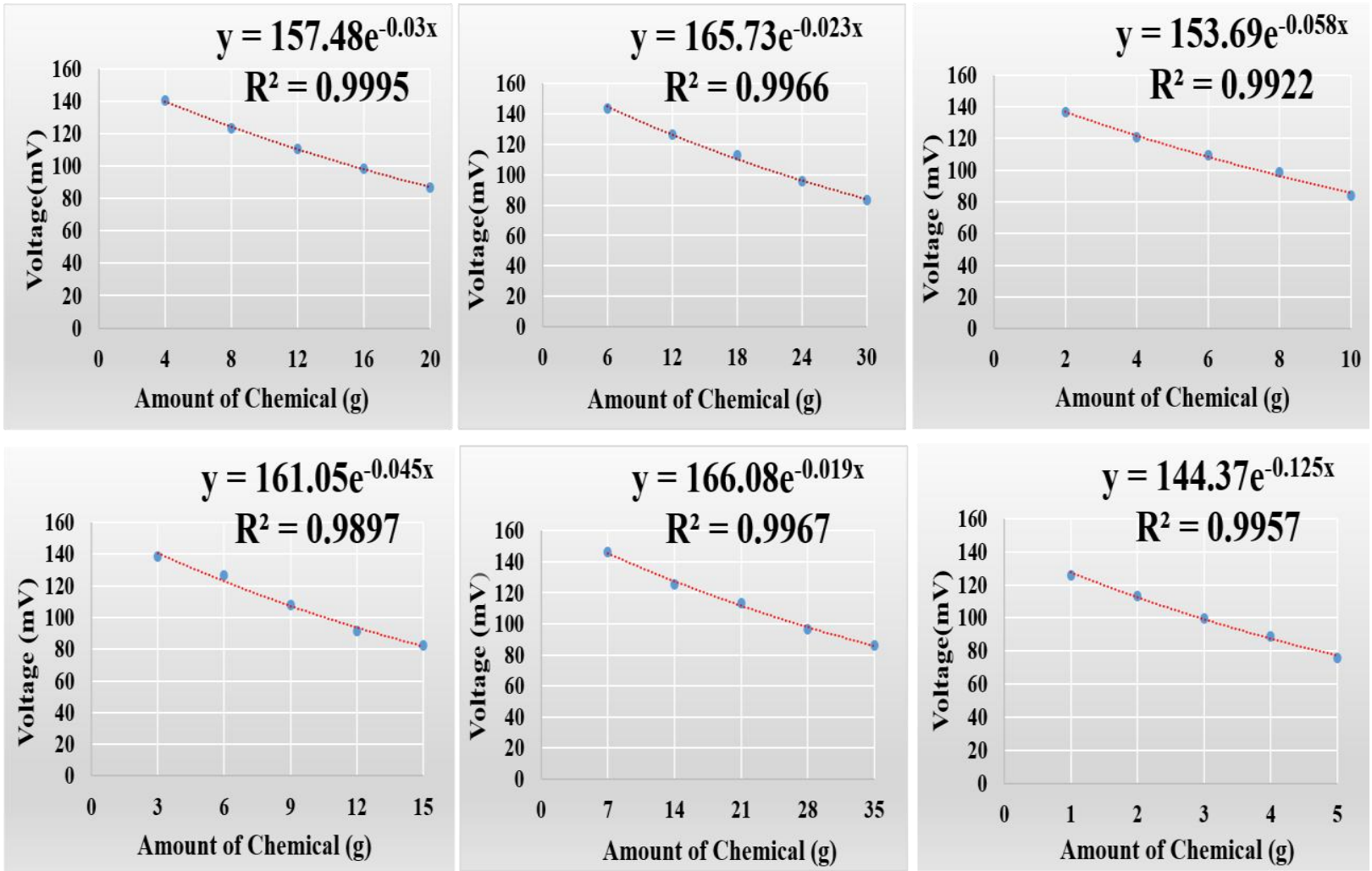

Figure 1: An empirical exponential based model based on soil reflectivity

\subsection{Monitoring the system through Arduino for Nitrogen content estimation}

The algorithm developed from the experimental data was fed into an arduino which forms the monitoring unit. The decision from which is displayed by the LCD which forms the display unit.Samples were tested with random amount of chemicals. The arduino monitors the system based on the algorithm so developed. Based on this, the results were displayed on an LCD. Real-time analysis will be possble though this system for extracting some information about the nitrogen content of a particular soil type. It will also be useful for an application which will be specific for a particular crop. The discrete arrangement so developed can be integrated into more robust systems and can be built into a more compact system.Figure 2 shows the experimental set-up of the aforementioned system.

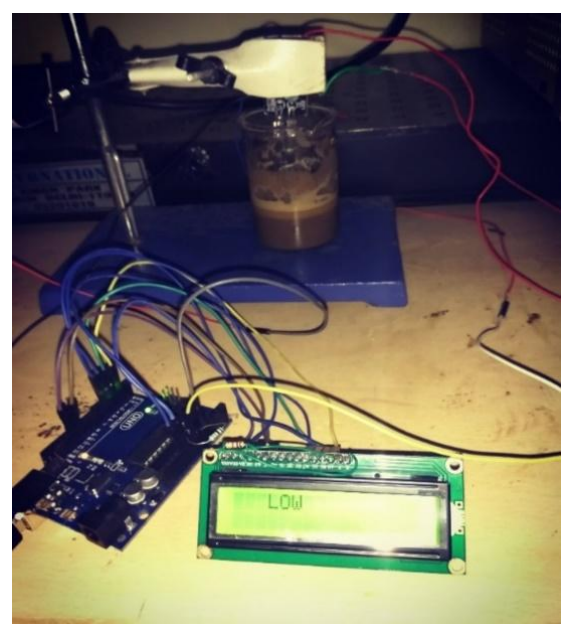

Figure 2: Set-up showing monitoring of Soil Nitrogen

\section{CONCLUSION}

Nitrogen in soil forms a major life-line when it comes to plant growth and fertility of the soil. Reflectance based studies have gained popularity while designing optical based sensor systems. In the present work, the potential of NIR based optical sensor in combination with regression analysis was studied for estimation of Nitrogen content in soil. Determining the right amount of nitrogen is important for improving $\mathrm{N}$-application management which can reduce soil and water pollution. The reflectivity of nitrogen in soil to an NIR sensor was studied and statistically analyzed. Based on these measurements, the system was represented mathematically using an exponential model. Calibration of a system is an important factor while designing and evaluating sensor systems. The sensor system so designed was calibrated successfully with a success rate of $90 \%$.Testing the system with raw data based on reflectivity was carried out by putting random data-points. The error analysis validated satisfactory results with RMSE of 0.5 while the $\mathrm{R}^{2}$ value close to 0.9 for almost all the combinations. Furthermore, an algorithm developed from the experimentation data was fed into an Arduino which forms the control and monitoring unit. The system so developed can influence precision farming in a better way. The methods implemented and the model so developed based on reflectivity measurements through voltage changes lays down a foundation for a cost-effective sensor design. The concept can further be extended for detecting nitrogen in other systems through intelligent setting of thresholds.

\section{ACKNOWLEDGEMENT}

Our thanks to the experts who have contributed significantly towards the successful design of the system. Firstly, we would like to thank Professor Shakuntala Laskar, HOD, Electrical and Electronics Department, ADBU for her help 
and support. Secondly, we would like to express our profound gratitude and regards to Professor Sunandan Baruah, HOD, ECE department, ADBU for giving valuable ideas and help.Thirdly, we would like to extend our gratitude to the Horticulture Research Institute and KVK, Kamrup (AAU) for their kind cooperation and help. We are highly obliged to Dr. Nirupoma Gogoi Baruah, Principal Scientist, Soil Science HRI, AAU, Kamrup and Goutami Kakati, Subject Matter Specialist, Soil Science, KVK, AAU, Kamrup for their valuable help provided in their respective field.

\section{REFERENCES}

[1] Bah, A and S.K. Balasundram, "Sensor Technologies for Precision Soil Nutrient Management and Monitoring", 2012, American Journal of Agricultural and Biological Sciences 7 (1): 43-49 ISSN 1557-4989

[2] V.I Adamchuk, J.W Hummel, M.T Morgan, S.K Upadhyaya, "On-the-go soil sensors for precision agriculture", Computers and Electronics in Agriculture, Volume 44, Issue 1, July 2004, Pages 7191,ISSN01681699,http://dx.doi.org/10.1016/j.compag.20 04.03.002.(http://www.sciencedirect.com/science/article/ pii/S0168169904 000444)

[3] J. A. Thomasson, R. Sui, M. S. Cox, A. Al Rajehy, "Soil Reflectance Sensing For Determining Soil Properties In Precision Agriculture", Transactions of the ASAE,Vol. 44(6) pages: 1445-1453@2001 American Society of Agricultural Engineers ISSN 0001-235

[4] Yubing Wang, Tianyu Huang, Jing Liu, Zhidan Lin, Shanhong Li, Rujing Wang, Yunjian Ge, "Soil pH value, organic matter and macronutrients contents prediction using optical diffuse reflectance spectroscopy", Computers and Electronics in Agriculture, 111 (2015) Pages: 69-77. (OElsevier BV

[5] Yandan Qiao and Shujuan Zhang, "Near-infrared spectroscopy technology for Soil nutrients detection based on LS-SVM", CCTA (1), volume 368 of IFIP Advances in Information and Communication Technology, page: 325-335. Springer, (2011)

[6] Michael Schirrmann, Robin Gebbers, Eckart Kramer and Jan Seidel, "Soil $\mathrm{pH}$ mapping with an On-The-Go Sensor", Sensors 2011, 11, pages: 573-598; doi: $10.3390 / \mathrm{s} 110100573$

[7] Flávio Anastácio de Oliveira Camargo, Clesio Gianello, Marino José Tedesco, João Riboldi ,Egon José Meurer ,Carlos Alberto Bissani, "Empirical models to predict soil nitrogen mineralization", ISSN 0103-8478, Ciência Rural, Santa Maria, v.32, n.3, pages:93-399, 2002

[8] Siti Jahara Matlan, Muhammad Mukhlisin and Mohd Raihan Taha "Performance Evaluation of FourParameter Models of the Soil-Water Characteristic Curve", Hindawi Publishing Corporation, The Scientific World Journal, Volume 2014, Article ID 569851, 12 pages http://dx.doi.org/10.1155/2014/569851

[9] Sophie Fabre, Xavier Briottet and Audrey Lesaignoux, "Estimation of Soil Moisture Content from the Spectral Reflectance of Bare Soils in the 0.4-2.5 $\mu \mathrm{m}$ Domain", Sensors 2015, 15, pp:3262-3281; doi:10.3390/s150203262, ISSN 1424-8220, www.mdpi.com/journal/sensors

[10] Lanfa Liu, Min Ji, Yunyun Dong, Rongchung Zhang and Manfred Buchroithne,"Quantitative Retrieval of Organic Soil Properties from Visible Near-Infrared Shortwave Infrared (Vis-NIR-SWIR) Spectroscopy Using FractalBased Feature Extraction", Remote Sens. 2016, 8, 1035; doi:10.3390/rs8121035

[11] Marek Wójtowicz ,Andrzej Wójtowicz ,Jan Piekarczyk "Application of remote sensing methods in agriculture", International Journal Of The Faculty Of Agriculture And Biology, Warsaw University Of Life Sciences ,Sggw, Poland, Vol. 11, No. 1, 2016, Pp. 31-50 @ CBCS

[12] Jardes Bragagnolo et al., "Optical crop sensor for variable-rate nitrogen fertilization in Corn: I-plant nutrition and dry matter production", 2013, R. Bras. Ci solo, pages: $1288-1298$

[13] T.N. Nath, "Status of Macronutrients (N, P And K) In Some Selected Tea Growing Soils Of Sivasagar District Of Assam, India " ,International Research Journal Of Chemistry (IRJC),ISSN,pages:2321 - 2845(Online), 2321 - 3299 (Print)

[14] Siti Noor Aliah Baharom, Sakae Shibusawa, Maskazu kodaira and Ryuhei Kanda, "Multiple-depth mapping of soil properties using visible and near infrared real time soil sensor for a paddy field", Engineering in Agriculture, Environment and Food ,8(2015) pages:1317 CElsevier.

[15] R. Sui, J. A. Thomasson, "Ground-Based Sensing System for Cotton Nitrogen Status Determination, Transactions of the ASABE Vol. 49(6) pages: 1983-1991@2006 American Society of Agricultural and Biological Engineers ISSN 0001-2351. 